\title{
THE IMAGE OF $\mathfrak{u}_{*}(X) \rightarrow \mathfrak{I}_{*}(X)$
}

GEORGE E. MITCHELL

In [3] Milnor characterized those bordism classes in the Thom bordism ring $\mathfrak{Y}_{*}$ which contain complex manifolds. ${ }^{1}$ This was done by showing that the image of the weakly complex bordism ring $u_{*}$ under the natural ring homomorphism $j: \mathfrak{U}_{*} \rightarrow \mathfrak{I}_{*}$ is precisely $\left(\mathfrak{I}_{*}\right)^{2}$. This paper is devoted to an extension of the above result. That is, the image of the natural homomorphism $j_{*}: \mathfrak{U}_{*}(X) \rightarrow \mathfrak{N}_{*}(X)$ is computed for certain spaces $X$.

Conner and Floyd [1, p. 45] defined the Whitney numbers of an element $\left[M^{n}, f\right]_{2} \in \mathscr{N}_{n}(X)$ as follows: Let $\sigma_{n}$ denote the fundamental classes of $M^{n}, w_{1}, \ldots, w_{n}$ denote the Stielfel-Whitney classes of $M^{n}$, and let $h^{m} \in H^{m}\left(X ; Z_{2}\right)$. For every partition $i_{1}+\cdots+i_{k}=n-m$ the number defined by

$$
\left\langle w_{i} \cdot \ldots \cdot w_{i_{k}} \cdot f^{*}\left(h^{m}\right), \sigma_{n}\right\rangle \in Z_{2}
$$

is called the Whitney number of $\left[M^{n}, f\right]_{2}$ associated with $h^{m}$. (Here $\langle$,$\rangle denotes the Kronecker product.) For X$ a CW-complex of finite type these Whitney numbers characterize the elements of $\Re_{*}(X)$.

For $X$ a CW-complex of finite type let $\left\{c_{n, i}\right\}$ be an additive basis for $H_{n}\left(X ; Z_{2}\right)$ for each $n$. Since the natural homomorphism $\mu: \mathfrak{N}_{n}(X)$ $\rightarrow H_{n}\left(X ; Z_{2}\right)$ defined by

$$
\mu\left(\left[M^{n}, f\right]_{2}\right)=f_{*}\left(\sigma_{n}\right)
$$

is onto, we may select a singular manifold $f_{i}: M_{i}^{n} \rightarrow X$ with $f_{i_{*}}\left(\sigma_{n}\right)=c_{n, i}$. Define an $\mathfrak{N}_{*}$-homomorphism $h: H_{*}\left(X ; Z_{2}\right) \otimes \mathscr{N}_{*} \rightarrow \mathfrak{N}_{*}(X)$ by $h\left(c_{n, i} \otimes 1\right)=\left[M_{i}^{n}, f_{i}\right]_{2}$. For $X$ a CW-complex of finite type $h$ is an isomorphism. (See $[1,(17.1)]$.)

Now let $X$ be a $C W$-complex for which the $\mathfrak{u}_{*}$-spectral sequence collapses. By $[2,(1.5)]$ we have that the natural map $\mathfrak{u}_{*}(X)$ $\rightarrow H_{*}(X ; Z)$ is an epimorphism. Let $\beta_{*}: H_{*}(X ; Z) \rightarrow H_{*}\left(X ; Z_{2}\right)$ be the reduction $\bmod 2$ and let $W$ be the image of $\beta_{*}$ in $H_{*}\left(X ; Z_{2}\right)$. Let $\left\{c_{q, i}\right\}$ be a homogeneous basis for $W$. ( $q$ denotes the dimension of $\left.c_{q, i}\right)$ Extend this basis to a basis of $H_{*}\left(X ; Z_{2}\right)$ by adding homogeneous basis elements $\left\{\tilde{c}_{q, j}\right\}$. All of the elements $c_{q, i}$ can be represented by weakly complex manifolds $V_{i}^{a}$ and maps $f_{i}: V_{i}^{a} \rightarrow X$ such that

Received by the editors, May 25, 1969.

AMS subject classifications. Primary 5530, 5560, 5710.

1 All manifolds are assumed to be smooth, compact and without boundary. 
$\beta_{*}\left(f_{i_{*}}\left(\sigma_{q, i}^{\prime}\right)\right)=c_{q, i}$, where $\sigma_{q, i}^{\prime} \in H_{q}\left(V_{i}^{q} ; Z\right)$ is the orientation class of $V_{i}^{q}$. For the basis elements $\tilde{c}_{q, j}$ we pick (nonweakly complex) manifolds $M_{j}^{q}$ and maps $f_{j}: M_{j}^{g} \rightarrow X$ such that $f_{j_{*}}\left(\sigma_{q, j}\right)=c_{q, j}$, where $\sigma_{q, j}$ $\in H_{q}\left(M_{j}^{q} ; Z_{2}\right)$ is the fundamental class.

Let $\left\{c^{q, i}\right\} \cup\left\{\tilde{c}^{q, j}\right\} \subset H^{*}\left(X ; Z_{2}\right)$ be a dual basis for $H^{*}\left(X ; Z_{2}\right)$ such that

$$
\begin{array}{ll}
\left\langle c^{q, i}, c_{q, i}\right\rangle=1=\left\langle\tilde{c}^{q, j}, \tilde{c}_{q, j}\right\rangle & \text { for all } i \text { and } j, \\
\left\langle c^{q, i}, c_{q, i^{\prime}}\right\rangle=0=\left\langle\tilde{c}^{q, j}, \tilde{c}_{q, j^{\prime}}\right\rangle & \text { for } i \neq i^{\prime} \text { and } j \neq j^{\prime}, \text { and } \\
\left\langle\tilde{c}^{q, j}, c_{q, i}\right\rangle=0=\left\langle c^{q, i}, \tilde{c}_{q, j}\right\rangle & \text { for all } i \text { and } j .
\end{array}
$$

Let $W^{\prime} \subset H^{*}\left(X ; Z_{2}\right)$ be the subspace spanned by $\left\{\tilde{c}^{q, j}\right\}$. Note that $W^{\prime}$ consists of exactly those elements $x \in H^{*}\left(X ; Z_{2}\right)$ for which $\left\langle x, \operatorname{Im} \beta_{*}\right\rangle=0$.

THEOREM 1. Let $X$ be a $\mathrm{CW}$-complex of finite type for which the $\mathfrak{u}_{*}$ spectral sequence collapses. If $\left[V^{n}, f\right]_{2}$ is the image of $\left[V^{n}, f\right]$ under the natural map $j_{*}: \mathcal{U}\left({ }_{*} X\right) \rightarrow \mathfrak{T}_{*}(X)$, then all the Whitney numbers of $\left[V^{n}, f\right]_{2}$ involving an odd $w_{i}$ (that is, $i$ is odd) or a homogeneous element $x \in W^{\prime}$ are zero.

Proof. The first part follows from the fact that the odd StiefelWhitney classes of a weakly complex manifold are all zero.

Suppose $x$ is a homogeneous element of $W^{\prime}$. Consider any Whitney number $\left\langle w_{2 i_{1}} \cdot \ldots \cdot w_{2 i_{k}} \cdot f^{*}(x), \sigma_{n}\right\rangle$. Here $\sigma_{n}$ is the mod 2 reduction of the orientation class $\sigma_{n}^{\prime} \in H_{n}\left(V^{n} ; Z\right)$; that is, $\beta_{*}\left(\sigma_{n}^{\prime}\right)=\sigma_{n}$. Since the even dimensional Stiefel-Whitney classes are the mod 2 reduction of the Chern classes [3] we may write

$$
\begin{aligned}
\left\langle w_{2 i_{1}} \cdot \ldots \cdot w_{2 i_{k}} \cdot f^{*}(x), \sigma_{n}\right\rangle & =\left\langle\beta^{*}\left(c_{i_{1}}\right) \cdot \ldots \cdot \beta^{*}\left(c_{i_{k}}\right) \cdot f^{*}(x), \beta_{*}\left(\sigma_{n}^{\prime}\right)\right\rangle \\
& =\left\langle f^{*}(x),\left(\beta^{*}\left(c_{i_{1}}\right) \cdot \ldots \cdot \beta^{*}\left(c_{i_{k}}\right)\right) \cap \beta_{*}\left(\sigma_{n}^{\prime}\right)\right\rangle \\
& =\left\langle f^{*}(x), \beta_{*}\left(\left(c_{i_{1}} \cdots c_{i_{k}}\right) \cap \sigma_{n}\right)\right\rangle \\
& =\left\langle x, f_{*} \beta_{*}\left(\left(c_{i_{1}} \cdots c_{i_{k}}\right) \cap \sigma_{n}\right)\right\rangle \\
& =\left\langle x, \beta_{*} f_{*}\left(\left(c_{i_{1}} \cdots c_{i_{k}}\right) \cap \sigma_{n}\right)\right\rangle .
\end{aligned}
$$

(Here $c_{i_{1}}, \ldots, c_{i_{k}}$ are Chern classes of $V^{n}$.) The last term is zero, since $\left\langle x, \operatorname{Im} \beta_{*}\right\rangle=0$. The conclusion follows.

THEOREM 2. Let $X$ be a CW-complex of finite type. If all the Whitney numbers of $\left[M^{n}, f\right]_{2}$ involving an odd $w_{i}$ or a homogeneous element $x \in W^{\prime} \subset H^{*}\left(X ; Z_{2}\right)$ are zero, then $\left[M^{n}, f\right]_{2}$ is in the image of $j_{*}: \mathfrak{U}_{*}(X)$ $\rightarrow \mathscr{T}_{*}(X)$.

Proof. By $[1,(17.1)]$ we may write 


$$
\left[M^{n}, f\right]_{2}=\sum_{q, i}\left[V_{i}^{n-q}, f_{i}\right]_{2} \cdot\left[Q_{i}^{q}\right]_{2}+\sum_{q, j}\left[M_{j}^{n-q}, f_{j}\right]_{2} \cdot\left[B_{j}^{q}\right]_{2} .
$$

We will show by induction on $q$ that $\left[Q_{i}^{q}\right]_{2}$ contains a weakly complex manifold and that $\left[B_{j}^{q}\right]_{2}=0$. Suppose true for $q<q_{0}$, and consider the Whitney number

$$
\left\langle w_{i_{1}} \cdot \ldots \cdot w_{i_{k}} \cdot f^{*}\left(\tilde{c}^{n-q_{0}, j_{0}}\right), \sigma_{n}\right\rangle,
$$

where $i_{k}+\cdots+i_{k}=q_{0}$ are picked arbitrarily. By assumption this number is zero. Now

$$
\begin{aligned}
& \left\langle w_{i_{1}} \cdot \ldots \cdot w_{i_{k}} \cdot f^{*}\left(\tilde{c}^{n-q_{0}, j_{0}}\right), \sigma_{n}\right\rangle \\
& =\sum_{q, i}\left\langle w_{i_{1}} \cdots \cdots w_{i_{k}} \cdot\left(f_{i} \pi\right)^{*}\left(\tilde{c}^{n-q_{0}, j_{0}}\right), \sigma_{n-q, i} \times \sigma_{q, i}\right\rangle \\
& \quad+\sum_{q, j}\left\langle w_{i_{1}} \cdots \cdots w_{i_{k}} \cdot\left(f_{j} \pi\right)^{*}\left(\tilde{c}^{n-q_{0}, j_{0}}\right), \sigma_{n-q, j} \times \sigma_{q, j}\right\rangle .
\end{aligned}
$$

All terms for which $q>q_{0}$ are zero by dimensional considerations. Since all the terms involving $q<q_{0}$ are Whitney numbers of weakly complex manifolds (by the induction hypothesis), they are also all zero. Hence this sum reduces to

$$
\begin{aligned}
& \sum_{i}\left\langle w_{i_{1}} \cdot \ldots \cdot w_{i_{k}} \cdot\left(f_{i} \pi\right)^{*}\left(\tilde{c}^{n-q_{0}, j_{0}}\right), \sigma_{n-q_{0}, i} \times \sigma_{q_{0}, i}\right\rangle \\
+ & \sum_{j}\left\langle w_{i_{1}} \cdot \ldots \cdot w_{i_{k}} \cdot\left(f_{j} \pi\right)^{*}\left(\tilde{c}^{n-q_{0}, j_{0}}\right), \sigma_{n-q_{0}, j} \times \sigma_{q_{0}, j}\right\rangle .
\end{aligned}
$$

All the terms of these sums are zero except for the term in which $j=j_{0}$. That is, the only nonzero term is

$$
\left\langle w_{i_{1}} \cdot \ldots \cdot w_{i_{k}} \cdot\left(f_{j_{0}} \pi\right)^{*}\left(\tilde{c}^{n-q_{0}, j_{0}}\right), \sigma_{n-q_{0}, j_{0}} \times \sigma_{q_{0}, j_{0}}\right\rangle .
$$

But this term is equal to the product

$$
\left\langle f_{j_{0}}^{*}\left(\tilde{c}^{n-q_{0}, j_{0}}\right), \sigma_{n-q_{0}, j_{0}}\right\rangle \cdot\left\langle v_{i_{1}} \cdot \ldots \cdot v_{i_{k}}, \sigma_{q_{0}, j_{0}}\right\rangle=\left\langle v_{i_{1}} \cdot \ldots \cdot v_{i_{k}}, \sigma_{q_{0}, j_{0}}\right\rangle,
$$

where the $v_{i}$ 's are the Stiefel-Whitney classes of $B_{j_{0}}^{q_{0}}$. But by assumption this Whitney number is zero for any partition $\left(i_{1}, \ldots, i_{k}\right)$ with $i_{1}+\cdots+i_{k}=q_{0}$ (since $\tilde{c}^{n-q_{0}, j_{0}}$ is in $W^{\prime}$ ). Hence $\left[B_{j_{0}}^{q_{0}}\right]_{2}=0$. Since $X$ is of finite type, there are only a finite number of choices for $j_{0}$. Hence the same argument shows that $\left[B_{j}^{q_{0}}\right]_{2}=0$ for all $j$.

Now consider the Whitney number

$$
\left\langle w_{i_{1}} \cdot \ldots \cdot w_{i_{k}} \cdot f^{*}\left(c^{n-q_{0}, i_{0}}\right), \sigma_{n}\right\rangle,
$$

where at least one of the $w_{i}$ 's has $i$ odd. By assumption this number is zero. The above argument shows that 


$$
\left\langle w_{i_{1}} \cdot \ldots \cdot w_{i_{k}} \cdot f^{*}\left(c^{n-q_{0}, i_{0}}\right), \sigma_{n}\right\rangle=\left\langle v_{i_{1}} \cdot \ldots \cdot v_{i_{k}}, \sigma_{q_{0}, i_{0}}\right\rangle,
$$

where the $v_{i}$ 's are the Stiefel-Whitney classes of $Q_{i_{0}}^{q_{0}}$. Since this number is zero for any partition $\left(i_{1}, \ldots, i_{k}\right)$ with $i_{1}+\cdots+i_{k}=q_{0}$ for which some $i_{r}(1 \leqq r \leqq k)$ is odd, it follows from Milnor [3] that $\left[Q_{i_{0}}^{q_{0}}\right]_{2}$ contains a weakly complex manifold.

We have shown that we may write

$$
\left[M^{n}, f\right]_{2}=\sum_{q, i}\left[V_{i}^{n-q}, f_{i}\right]_{2} \cdot\left[Q_{i}^{q}\right]_{2}
$$

where the $V_{i}^{n-q}$ are weakly complex manifolds and the $\left[Q_{i}^{q}\right]_{2}$ contain weakly complex manifolds. It follows that $\left[M^{n}, f\right]_{2}$ is in the image of $j_{*}: \mathfrak{u}_{*}(X) \rightarrow \mathfrak{T}_{*}(X)$. This completes the proof.

In conclusion we have proven the following:

TheOREM. For $X$ a CW-complex of finite type for which the $\mathfrak{u}_{*}$-spectral sequence collapses we have

$$
\operatorname{Im} j_{*}=h\left(\operatorname{Im} \beta_{*} \otimes\left(\mathscr{T}_{*}\right)^{2}\right) \subset \mathscr{N}_{*}(X),
$$

where $h$ is the isomorphism $H_{*}\left(X ; Z_{2}\right) \otimes \Re_{*} \rightarrow \mathscr{N}_{*}(X)$.

\section{REFERENCES}

1. P. E. Conner and E. E. Floyd, Differentiable periodic maps, Ergebnisse der Mathematik und ihrer Grenzgebiete, Band 33, Academic Press, New York and Springer-Verlag, Berlin and New York, 1964. MR 31 \#750.

2. - Torsion in SU-bordism, Mem. Amer. Math. Soc. No. 60 (1966). MR $32 \# 6471$.

3. J. W. Milnor, On the Stiefel-Whitney numbers of complex manifolds and of spin manifolds, Topology 3 (1965), 223-230. MR 31 \#5207.

4. N. Steenrod, The topology of fibre bundles, Princeton Math. Series, vol. 14, Princeton Univ. Press, Princeton, N. J., 1951. MR 12, 522.

West Virginia University, Morgantown, West Virginia 26506 